\title{
A Comparison of qSOFA and SIRS in the Early Diagnosis of Severe Pneumococcal Sepsis: A Retrospective Cohort Study
}

\author{
Pernille Ursin Rein ${ }^{1 *}$, Dag Jacobsen ${ }^{1}$, Vidar Ormaasen ${ }^{2}$, Vera Djordjilović ${ }^{3}$ and Oona Dunlop ${ }^{1}$ \\ ${ }^{1}$ Department of Acute Medicine, Oslo University Hospital Ullevål, Norway \\ ${ }^{2}$ Department of Infectious Diseases, Oslo University Hospital Ullevål, Norway \\ ${ }^{3}$ Department of Biostatistics, Oslo Centre for Biostatistics and Epidemiology, University of Oslo, Norway

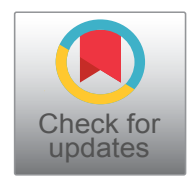

*Corresponding author: Pernille Ursin Rein, Resident in Anesthesiology, Department of Anesthesia, Oslo University Hospital Ullevål, PB 4956 Nydalen, 0424 Oslo, Norway, Tel: +47-97537061, Fax: +47-22117485

\begin{abstract}
Background: In 2016 quick Sequential [Sepsis-related] Organ Failure Assessment (qSOFA) was presented as a simple bedside screening tool in identifying sepsis and is now replacing the Systemic Inflammatory Response Syndrome (SIRS) criteria in many Emergency Departments. We wanted to study whether these diagnostic criteria were sensitive enough to identify sepsis on the initial hospital admission in a cohort of patients who rapidly developed pneumococcal septic shock.
\end{abstract}

Methods: We retrospectively studied qSOFA and SIRS criteria in a previously described cohort of all adult patients with severe pneumococcal sepsis admitted to the Medical Intensive Care Unit at Oslo University Hospital Ullevaal. This cohort consists of 38 patients that were included in an eightyear period (01/01/2006-12/31/2013). The inclusion criteria were growth of Streptococcus pneumoniae in blood culture and respiratory failure treated with invasive mechanical ventilation, thus fulfilling the definition of severe sepsis. Data to assess the sensitivity of the SIRS - and the qSOFA criteria on admission was available in 31 of 38 patients. Outcome was measured as number of patients that fulfilled the criteria for sepsis on admission, defined by the three following groups: SIRS $\geq 2 / 4$, SIRS $\geq 3 / 4$ and qSOFA $\geq 2 / 3$.

Results: The qSOFA criteria $\geq 2 / 3$ identified $71 \%$ of the patients, $\geq 2 / 4$ SIRS criteria identified $97 \%$ and $\geq 3 / 4$ SIRS criteria identified $87 \%$ of the patients on admission. The sensitivity of qSOFA criteria $\geq 2 / 3$ was lower than SIRS criteria $\geq 2 / 4$ (statistically significant, $p=0.003$ ). The use of both qSOFA and SIRS ( $\geq 2 / 4$ or $\geq 3 / 4$ ) identified sepsis in all patients on admission.

Conclusion: In this cohort of untreated patients at admission, who rapidly developed pneumococcal septic shock, the qSOFA score on admission failed to identify sepsis in almost one-third of the septic patients and was not sensitive enough to be used alone in our population.

\section{Keywords}

Sepsis, qSOFA, Systemic inflammatory response syndrome, Streptococcus pneumoniae, Septic shock, Screening tool

\begin{abstract}
Abbreviations
CBRNE: Chemical-, Biological-, Radioactive-, Nuclearand Explosive agents; ED: Emergency Department; GCS: Glasgow Coma Scale; CSF: Cerebrospinal Fluid; ICU: Intensive Care Unit; MICU: Medical Intensive Care Unit; OUH: Oslo University Hospital; qSOFA: quick Sequential [Sepsis-related] Organ Failure Assessment; SIRS: Systemic Inflammatory Response Syndrome; SOFA: Sequential [Sepsis-related] Organ Failure Assessment
\end{abstract}

\section{Introduction}

Sepsis criteria are important: As bedside diagnostic tools to identify septic patients, as disease-defining criteria in research and finally in severity scoring/mortality prediction. The Systemic Inflammatory Response Syndrome (SIRS) criteria were introduced in 1992 [1]. Later attempts to implement other sepsis criteria have been unsuccessful [2].

In 2016 Sepsis-3 was presented; Sequential [Sepsis-related] Organ Failure Assessment (SOFA) and quickSOFA (qSOFA) scores were defined as the new sepsis criteria instead of SIRS [3]. The major change was from

Citation: Rein PU, Jacobsen D, Ormaasen V, Djordjilović V, Dunlop O (2020) A Comparison of qSOFA and SIRS in the Early Diagnosis of Severe Pneumococcal Sepsis: A Retrospective Cohort Study. Int J Anesthetic Anesthesiol 7:111. doi.org/10.23937/2377-4630/1410111

Accepted: August 08, 2020: Published: August 10, 2020

Copyright: (C) 2020 Rein PU, et al. This is an open-access article distributed under the terms of the Creative Commons Attribution License, which permits unrestricted use, distribution, and reproduction in any medium, provided the original author and source are credited. 
a focus on inflammation (SIRS) to organ dysfunction (SOFA) as a result of the dysregulated host response to infection. Several studies have shown a good correlation between SOFA/qSOFA and in-hospital mortality [4-7]. In many Emergency Departments (EDs) qSOFA is now about to replace the SIRS criteria as a simple bedside diagnostic tool [8]. However, the value of qSOFA to make an early diagnosis of sepsis has been questioned in several studies [9-12].

Identifying the septic patient in the early phase can be difficult, even for the experienced clinician. The combination of a heterogeneous clinical picture and the lack of specific laboratory parameters make the initial diagnosis a challenge. Good diagnostic tools in the evaluation of patients can therefore support clinicians where sepsis may be a differential diagnosis. After decades with non-conclusive studies on possible new therapeutic measures in treating sepsis, we are left with supportive care and especially prompt administration of antibiotics where time is critical for survival [13-16].

There are several large multicenter studies evaluating qSOFA in the emergency department both as a simple bedside diagnostic tool and also as a mortality predictor $[6,17]$. The patients in these studies often represent a heterogeneous group regarding bacteriological findings and severity of disease. There are few publications of studies that consist solely of the most severe cases of sepsis. The situation that represents the largest challenge to the bedside diagnostics are patients that rapidly decline to septic shock, i.e. septic patients with a narrow time-gap to initiate treatment that may improve the course of disease.

We have previously described a rare, homogeneous patient cohort with severe pneumococcal sepsis and rapid development of septic shock [18]. This cohort is of particular interest since it includes all eligible patients, none had received antibiotics before hospital admission, the diagnosis of sepsis was at this point not yet confirmed and they rapidly developed septic shock and multiorgan failure. The aim of the present study was to retrospectively compare the sensitivity of qSOFA to SIRS criteria in diagnosing sepsis on the primary admission in the ED in this cohort.

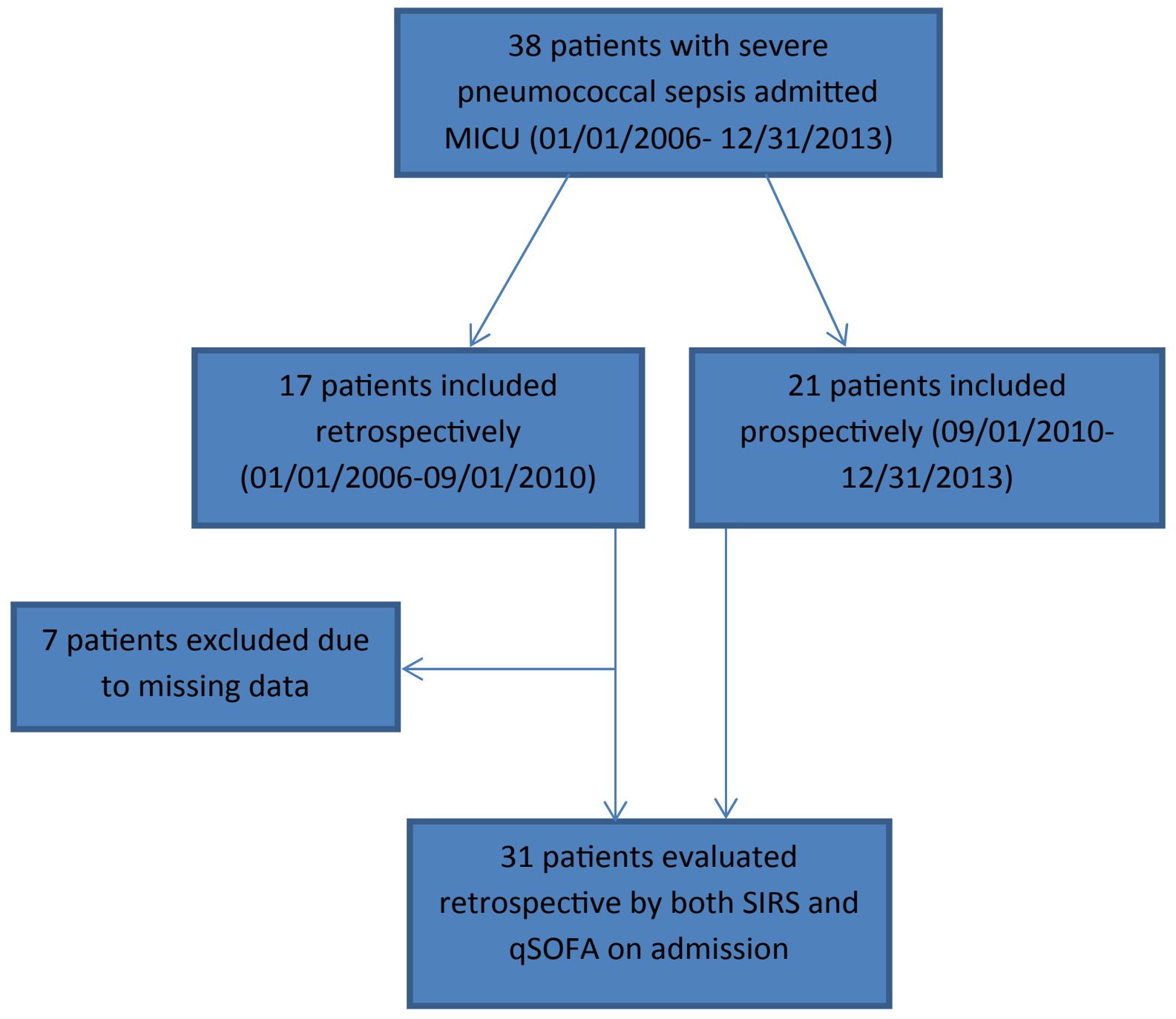

Figure 1: Flow-chart of the included patients in the study. The inclusion criteria of the original cohort of 38 patients were growth of Streptococcus pneumoniae in blood culture and respiratory failure, defined as the need for mechanical ventilation. Note that all patients progressed to septic shock. 


\section{Methods}

\section{Sepsis and SIRS-/qSOFA criteria}

We used the international SIRS criteria originally defined by the American College of Chest Physicians/ Society of Critical Care Medicine consensus conference committee [1] and the qSOFA criteria that was presented in 'Sepsis-3' [3]. SIRS criteria: Temperature $>38^{\circ} \mathrm{C}$ or $<36{ }^{\circ} \mathrm{C}$; heart rate $>90$ beats per minute; respiratory rate $>20$ breaths per minute or $\mathrm{PaCO}_{2}<4.3 \mathrm{kPa}$; white blood cell count $>12.0 \times 10^{9} / \mathrm{L}$ or $<4.0 \times 10^{9} / \mathrm{L}$ or $>10 \%$ immature bands. qSOFA criteria: Respiratory rate $\geq 22$ breaths per minute; altered mentation (Glasgow Coma Scale (GCS) < 15); systolic blood pressure $\leq 100 \mathrm{mmHg}$. In both, the patients fulfill the sepsis definition if they have $\geq 2$ criteria upon admission in combination with a suspected infection (SIRS criteria and qSOFA score is given in (Figure 2). We also included SIRS $\geq 3 / 4$ which has been used in larger controlled sepsis trials $[19,20]$.

We used the definitions for severe sepsis and septic shock that were defined together with the SIRS criteria in 1992; severe sepsis was defined as sepsis associated with organ dysfunction, hypoperfusion or hypotension and septic shock was defined as the need for treatment with vasopressor despite adequate fluid resuscitation [1].

\section{Design and data collection - original cohort and present study}

We retrospectively studied the ability of qSOFA and SIRS criteria to identify sepsis at admission in a previously described cohort of all eligible adult patients with severe pneumococcal sepsis (38 patients) admitted to the Medical Intensive Care Unit (MICU) at Oslo University Hospital (OUH) Ullevaal in an eight-year period (01/01/2006-12/31/2013) [18]. The study design was combined retrospective (01/01/2006-09/01/2010) and prospective (09/01/2010-12/31/2013). The population was sampled by a manual review of the MICUs patient registry. The inclusion criteria in this cohort were respiratory failure, defined as treatment with invasive mechanical ventilation, and growth of Streptococcus pneumoniae in blood cultures (confirmation of bacteriology). Thus the inclusion criteria fulfill the definition of severe sepsis. However, all patients also rapidly developed multiorgan failure and septic shock, the majority within 24 hours from their first medical evaluation, and none had received antibiotics before admission. The focus of the original paper was to study the course of the most severe cases of pneumococcal sepsis.

In the present study we retrospective evaluated the SIRS and qSOFA criteria upon the primary admission. Patients from the original cohort were included in this study if all criteria for defining both qSOFA and SIRS were recorded at the primary admission in the ED. Patients were excluded if one or more of the given criteria were not re- corded. Data to assess the sensitivity of qSOFA- and SIRS criteria were collected from the admission journal and - chart [18]. For those patients who were transferred from their local hospital, the data were collected from the primary evaluation at the respective hospitals. As such, all data were from the initial hospitalization. Note that the focus of the present retrospective study is on the ability of the considered criteria to identify sepsis on admission in patients who all developed severe sepsis; as such its" design - in particular, the absence of "true negatives" and "false positives" - does not allow for the comparison of other performance measures such as positive and negative predictive value or specificity.

OUH Ullevaal is a university teaching hospital and tertiary care center that also has the function of a primary hospital and the patient population hence comprises of both selected and unselected groups of patients. The primary population consists of 220,000 people which is approximately $1 / 3$ of Oslo's citizens. The ED of OUH Ullevaal has approximately 30,000 admissions per year. The MICU has 12 beds and six mechanical ventilators. The patients admitted are critically ill patients mainly with infections, metabolic diseases and intoxications. The MICU has a close collaboration with the other specialized intensive care units (ICUs) at the hospital and works in close collaboration with the National Medical Centre of Chemical-, Biological-, Radioactive-, Nuclear- and Explosive agents (CBRNE). The staff consists of intensive care physicians, emergency medicine physicians, infectious disease specialists, anesthetists and others.

\section{Primary focus of infection}

An attempt to identify primary focus of infection was made. The assumption was based on clinical features, radiology and further examinations. Pneumonia was registered as the focus of infection when the radiologic examinations revealed pneumonic infiltrates. The diagnosis of meningitis was based on cerebrospinal fluid (CSF) findings with raised white blood cell count and in addition pathological CSF/glucose ratio, positive pneumococcal antigen test or positive microscopy with gram-positive diplococci in the CSF. The diagnosis of otitis was based on typical findings at otoscopy. Otitis was registered as the primary infection in the cases of otogenous meningitis.

\section{Original definitions for organ failure}

Circulatory failure or septic shock was defined as the need for treatment with vasopressor despite adequate fluid resuscitation [1]. Heart failure was arbitrarily defined as an ejection fraction $(\mathrm{EF})<30 \%$ verified by echocardiography. Respiratory failure was defined as the need of invasive mechanical ventilation. Kidney failure was defined as treatment with renal-replacement therapy, both continuous venovenous hemodialysis (CVVHD) and intermittent hemodialysis (IHD). Liver failure 


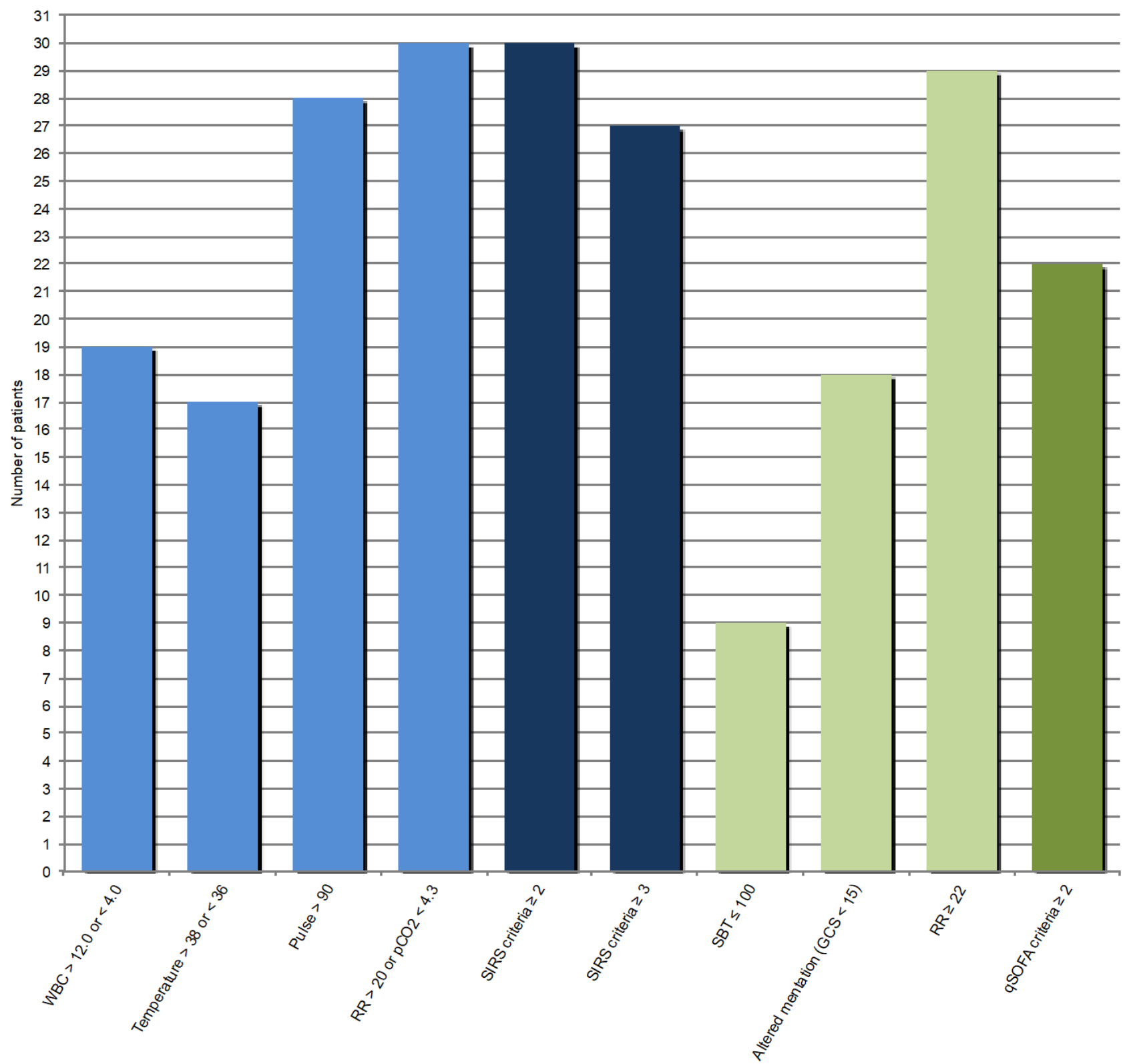

Figure 2: SIRS and qSOFA parameters on admission in 31 patients with pneumococcal septic shock. WBC: white blood cells, RR: respiratory rate, SBP: systolic blood pressure, GCS: Glasgow Coma Scale.

was defined as a hepatic SOFA score $\geq 3$ (bilirubin $\geq 102$ micromol/L) $[3,21]$. Disseminated intravascular coagulation (DIC) was defined as an International Society on Thrombosis and Haemostasis (ISTH) score $\geq 5$ [22], development of peripheral necrosis and/or microthrombosis verified by biopsy or autopsy.

\section{Outcome}

Outcome was measured as number of patients that fulfilled the criteria for sepsis on admission, defined by the three following groups: SIRS $\geq 2 / 4$, SIRS $\geq 3 / 4$ and qSOFA $\geq 2 / 3$. Clinical outcome was measured as death (in-hospital), surviving with severe sequelae or returning to habitual state. Severe sequelae were defined as amputation and/or adverse neurologic complications.

\section{Statistics}

To statistically evaluate the sensitivity of the three groups of diagnostic criteria, we obtained point estimates of the sensitivities and constructed the associated $95 \%$ asymptotic confidence intervals. To evaluate the statistical significance of the difference between the sensitivity of the qSOFA and the two SIRS groups of criteria, we performed the statistical tests of the hypotheses of their equality at the level of $5 \%$. We also computed the associated $95 \%$ confidence intervals for the differences in sensitivities.

\section{Ethics}

We obtained informed consent (written or oral) by the patients or their relatives. The study was approved by the Centre of Patient Privacy and Safety at 
OUH (protocol number 2012/11901, date of approval $07 / 26 / 2012$ ). Age was given in decades in order to prevent identification of individual patients (Table 1). All data were handled and saved anonymously.

\section{Results}

\section{Patients and initial presentation}

Seven patients (patients 1, 5, 6, 11, 12, 15, 16 in paper (18)) were excluded from the original cohort of 38 patients because of unregistered data. The excluded patients were one male (50 years) and 6 females (30-70 years), two of them died (females), one survived with amputations of all extremities and the other four had an uneventful recovery. Thus, the initial qSOFA - and SIRS

Table 1: Patient summary and clinical parameters.

\begin{tabular}{|c|c|c|c|c|c|c|c|}
\hline \multicolumn{5}{|c|}{ Admission data } & \multicolumn{3}{|c|}{ Disease progression } \\
\hline Pat. ${ }^{1}$ & $\begin{array}{l}\text { Gender - } \\
\text { Age }\end{array}$ & SIRS $^{2}$ & qSOFA $^{3}$ & Focus $^{4}$ & DIC & MOF $^{5}$ & $\begin{array}{l}\text { Outcome }^{6} \\
\text { (Days until death) }\end{array}$ \\
\hline 2 & M 70 & $3 / 4 p, r, t$ & $1 / 3 \mathrm{~m}$ & $M$ & + & $4 / 6$ & Dead (20) \\
\hline 3 & F 50 & $2 / 4 p, r$ & $3 / 3$ & $\mathrm{P}$ & + & $4 / 6$ & Dead (9) \\
\hline 4 & M 40 & $3 / 4 p, r, w$ & $1 / 3 r$ & $\mathrm{P}$ & - & $4 / 6$ & Recovery \\
\hline 7 & F 50 & $3 / 4 p, r, w$ & $1 / 3 r$ & $\mathrm{P}$ & - & $2 / 6$ & Recovery \\
\hline 8 & M 70 & $4 / 4$ & $2 / 3 \mathrm{~m}, \mathrm{r}$ & $P$ & + & $5 / 6$ & Dead (20) \\
\hline 9 & F 50 & $3 / 4 p, r, t$ & $2 / 3 \mathrm{~m}, \mathrm{r}$ & $\mathrm{U}$ & + & $4 / 6$ & Dead (97) \\
\hline 10 & M 40 & $3 / 4 p, r, w$ & $2 / 3 b, r$ & $U$ & + & $5 / 6$ & Sequela $(A)$ \\
\hline 13 & M 50 & $3 / 4 p, r, w$ & $1 / 3 r$ & $\mathrm{P}$ & - & $3 / 6$ & Recovery \\
\hline 14 & M 20 & $3 / 4 \mathrm{p}, \mathrm{r}, \mathrm{w}$ & $2 / 3 b, r$ & $P$ & - & $2 / 6$ & Recovery \\
\hline 17 & M 60 & $3 / 4 p, r, t$ & $2 / 3 \mathrm{~m}, \mathrm{r}$ & $\mathrm{O}(\mathrm{M})$ & - & $2 / 6$ & Recovery \\
\hline 18 & F 50 & $3 / 4 p, r, t$ & $1 / 3 r$ & $\mathrm{M}$ & + & $4 / 6$ & Dead (99) \\
\hline 19 & M 20 & $4 / 4$ & $2 / 3 \mathrm{~m}, \mathrm{r}$ & $M$ & - & $2 / 6$ & Sequela $(N)$ \\
\hline 20 & M 70 & $3 / 4 p, r, w$ & $2 / 3 b, r$ & $P$ & - & $4 / 6$ & Dead (2) \\
\hline 21 & F 60 & $3 / 4 p, r, t$ & $2 / 3 \mathrm{~m}, \mathrm{r}$ & M & + & $4 / 6$ & Dead (67) \\
\hline 22 & F 50 & $3 / 4 \mathrm{p}, \mathrm{r}, \mathrm{w}$ & $2 / 3 b, r$ & $P$ & + & $4 / 6$ & Recovery \\
\hline 23 & F 60 & $3 / 4 \mathrm{p}, \mathrm{r}, \mathrm{w}$ & $1 / 3 r$ & $P$ & - & $2 / 6$ & Recovery \\
\hline 24 & M 70 & $2 / 4 r, w$ & $2 / 3 \mathrm{~m}, \mathrm{r}$ & $\mathrm{P}$ & - & $2 / 6$ & Dead (27) \\
\hline 25 & M 50 & $3 / 4 p, r, w$ & $2 / 3 \mathrm{~b}, \mathrm{r}$ & $P$ & - & $2 / 6$ & Recovery \\
\hline 26 & F 30 & $3 / 4 p, r, t$ & $1 / 3 r$ & $P$ & - & $2 / 6$ & Recovery \\
\hline 27 & M 40 & $4 / 4$ & $1 / 3 r$ & $P$ & - & $3 / 6$ & Dead (51) \\
\hline 28 & F 50 & $4 / 4$ & $1 / 3 r$ & $\mathrm{P}$ & - & $2 / 6$ & Dead (35) \\
\hline 29 & M 60 & $3 / 4 p, r, t$ & $2 / 3 \mathrm{~m}, \mathrm{r}$ & $P$ & - & $2 / 6$ & Recovery \\
\hline 30 & F 60 & $3 / 4 p, r, t$ & $2 / 3 \mathrm{~m}, \mathrm{r}$ & $P(M)$ & - & $3 / 6$ & Sequela $(N)$ \\
\hline 31 & F 50 & $3 / 4 p, r, w$ & $3 / 3$ & $\mathrm{O}(\mathrm{M})$ & - & $2 / 6$ & Sequela $(N)$ \\
\hline 32 & M 40 & $1 / 4 w$ & $2 / 3 b, m$ & $\mathrm{M}$ & - & $2 / 6$ & Dead (2) \\
\hline 33 & F 70 & $3 / 4 p, r, t$ & $2 / 3 \mathrm{~m}, \mathrm{r}$ & $U$ & + & $4 / 6$ & Sequela $(A)$ \\
\hline 34 & M 50 & $3 / 4 p, r, t$ & $3 / 3$ & $\mathrm{U}$ & + & $4 / 6$ & Dead (79) \\
\hline 35 & M 70 & $4 / 4$ & $2 / 3 \mathrm{~m}, \mathrm{r}$ & $\mathrm{O}(\mathrm{M})$ & - & $3 / 6$ & Dead (9) \\
\hline 36 & M 40 & $4 / 4$ & $2 / 3 \mathrm{~m}, \mathrm{r}$ & $\mathrm{O}(\mathrm{M})$ & - & $2 / 6$ & Recovery \\
\hline 37 & M 70 & $2 / 4 p, r$ & $2 / 3 \mathrm{~m}, \mathrm{r}$ & $\mathrm{P}$ & + & $4 / 6$ & Sequela $(A+N)$ \\
\hline 38 & F 60 & $3 / 4 r, t, w$ & $2 / 3 \mathrm{~m}, \mathrm{r}$ & M & - & $2 / 6$ & Sequela $(N)$ \\
\hline
\end{tabular}

1)Pat: Patient. Patient numbers are unchanged from original study (1-38)(18).

2)SIRS; w: Positive score white blood cell count, p: Positive score pulse rate, t: Positive score temperature, r: Positive score respiration.

${ }^{3)}$ qSOFA; r: Positive score respiration, b: Positive score blood pressure, m: Positive score altered mentation (GCS < 15). See Figure 2 for definitions.

4) P: Pneumonia; O: Otitis; M: Meningitis; O (M): Meningitis secondary to otitis; U: Unknown.

${ }^{5}$ MOF: Multiorgan failure. All patients developed multiorgan failure with respiratory (inclusion criterion) and circulatory failure (septic shock) resulting in minimum 2/6 (two out of six possible organ failures). DIC: disseminated intravascular coagulation;

${ }^{6} \mathrm{~N}$ : Neurological sequelae; A: Amputated. 
score was retrospectively evaluated, upon the primary hospital admission, in 31 patients.

The majority of the patients had OUH Ullevaal as their primary hospital. Eleven of the patients were transferred to us (tertiary care center) because of the need for more specialized treatment. Of the 31 patients, 18 were men and 13 women Table 1 . The median age was 59 years (range $22-79$ ).

The patients were registered in the ED with a variety of admitting diagnoses. Most of the patients were in retrospect assumed to have pneumonia as their primary infection (17/31; 55\%). Eleven of the patients (35\%) had meningitis, primary or secondary. Other primary foci are given in (Table 1). All patients developed septic shock and in 26/31 (84\%) it took less than 24 hours from the first medical evaluation until admission in the MICU with fully developed septic shock. No patients received antibiotics before hospital admission.

\section{SIRS and qSOFA}

The number of positive SIRS - and qSOFA criteria for each patient are given in Table 1. For the SIRS criteria the respective values were: Respiration 30/31 (97\%), pulse 28/31 (90\%), temperature 17/31 (55\%), white blood cell (WBC) count 19/31 (61\%). For the qSOFA criteria the respective values were: respiration $29 / 31$ (94\%), altered mentation 18/31 (58\%), hypotension 9/31 (29\%). At the time of hospitalization, 22/31 patients fulfilled the qSOFA ( $\geq 2 / 3$ ) criteria (estimated sensitivity $71 \%$ with the $95 \%$ confidence interval $(55 \%, 87 \%)) ; 30 / 31$ patients fulfilled the SIRS criteria ( $\geq 2 / 4$ ) (estimated sensitivity $97 \%$ with the $95 \%$ confidence interval $(91 \%, 100 \%))$; 27/31 patients fulfilled the SIRS (3/4) criteria (estimated sensitivity $87 \%$ with the $95 \%$ confidence interval (75\%, 99\%)) (Figure 2).

The few patients not diagnosed with sepsis by SIRS $\geq$ $2 / 4$ (patient 32) or $\geq 3 / 4$ (patients $3,24,32,37$ ) were all diagnosed by qSOFA $\geq 2 / 3$ (Table 1 ). As such, all patients were identified using qSOFA $(\geq 2 / 3)$ in combination with SIRS ( $\geq 2 / 4$ or $\geq 3 / 4$ ).

The observed difference between the sensitivity of qSOFA ( $\geq 2 / 3$ ) and SIRS ( $2 / 4)$ is $26 \%$ and it is statistically significant ( $p=0.003 ; 95 \%$ confidence interval for the difference is $(9 \%, 43 \%))$; the difference between the sensitivity of the qSOFA $(\geq 2 / 3)$ and SIRS $(\geq 3 / 4)$ is $16 \%$ and it is not statistically significant at the level of $5 \%$, i.e. $95 \%$ confidence interval for the difference is $(-4 \%, 36 \%)$.

\section{Multiorgan failure (MOF), morbidity and mortality}

Number of organ failures in each patient is given in (Table 1). Details regarding type of organ failures in the different patients and full SOFA scores during the stay at the MICU are presented elsewhere [18]. In-hospital mortality was $42 \%$ (13/31), $23 \%$ (7/31) of the patients survived with severe sequelae and $35 \%$ (11/31) returned to their habitual state (Table 1).

\section{Discussion}

qSOFA on admission missed to identify sepsis in almost one-third (9/31) of the patients in this cohort. In comparison, both the SIRS criteria $\geq 2 / 4$ and $\geq 3 / 4$ identified more patients, missing only one (1/31) and four (4/31) patients, respectively. The observed difference in sensitivity between qSOFA ( $\geq 2 / 3$ ) and SIRS ( $\geq 2 / 4)$ was statistically significant at the $5 \%$ level, indicating that qSOFA had lower sensitivity. Interestingly, the combined use of qSOFA and SIRS ( $\geq 2 / 4$ or $\geq 3 / 4$ ) identified sepsis in all patients on their first admission.

The clinical presentation in the population was quite variable and sepsis was seldom the admitting diagnosis [18]. Many had gastrointestinal features that mislead the clinicians, resembling gastroenteritis. As such, the clinical diagnosis was a challenge in these patients, where all rapidly developed septic shock. In such patients, it is of utmost importance to have a good diagnostic tool to aid early recognition of sepsis and start antibiotic therapy quickly.

In Sepsis-3 the task force presents qSOFA as simple bedside criteria that can be used not only as a mortality predictor but also as a screening tool for sepsis [3]. The SIRS criteria have been routinely used by physicians in identifying sepsis in many EDs, and after the publication of Sepsis-3 qSOFA has become an additional screening tool. The qSOFA criteria have been validated as a mortality predictor $[4,6]$. However, concerns have been raised to the diagnostic properties of the criteria and the importance of focus on early diagnosis rather than mortality prediction has been addressed $[23,24]$. Haydar, et al. retrospectively evaluated and compared qSOFA and SIRS criteria ( $\geq 2 / 4)$ at admission in the ED in 200 patients with sepsis and found a poor performance of the qSOFA criteria, identifying only $58 \%$ compared to 95\% of the patients using the SIRS criteria [25]. Our results are similar, but in-hospital mortality was $11 \%$ compared to $42 \%$ in our study. Askim, et al. also found a low sensitivity of qSOFA in a Norwegian ED though in a more heterogeneous sepsis population [9]. Presumably both qSOFA and SIRS criteria will become more sensitive during the progression of sepsis, i.e. it is more likely that the criteria will identify sepsis in the later phases of sepsis. The question is not whether the criteria could identify sepsis at all, but if they can identify sepsis in the early phases.

Of the different parameters, tachypnea was by far the most sensitive in both qSOFA and SIRS. More than half of the patients had altered mentation (GCS < 15) - a sign originally ascribed to (cerebral) hypoperfusion under the previous term "severe sepsis" [1]. Lack of consideration of mental status is a commonly agreed weakness of the SIRS criteria. One explanation of our high number of patients with altered mentation could be that one-third of the patients had septic shock originating from meningitis. On the other hand, as seen from 
(Table 1), nearly half of the patients with altered mentation did not have meningitis. This finding in our patients, support the inclusion of this parameter in the new sepsis definition [3].

The parameter responsible for the low sensitivity of qSOFA in this patient cohort was hypotension. Compensatory mechanisms make hypotension a late clinical sign of evolving or established cardiovascular dysfunction. In young and healthy individuals adrenergic countermeasures may postpone the development of hypotension, but when it finally occurs the patient is already in septic shock with a far more severe prognosis. As indicated in our group of patients, this may weaken the sensitivity of the qSOFA in the early phase of the most severe cases of sepsis.

\section{Limitations}

Our study has several limitations. First, it is a small cohort from a single-center and the design is retrospective. Second, the patient group is highly selected - they all had pneumococcal sepsis, respiratory failure and were admitted to the MICU at OUH Ullevaal. In addition, the cohort turned out to be more homogenous than expected as all rapidly developed septic shock with multiorgan failure. The patients therefore do not represent septic patients in general. The high proportion of patients with meningitis may have contributed to a higher qSOFA score.

Despite the limitations we would like to highlight the selection of the cohort, which is in fact one of the largest published of its kind $[18,26]$. Sepsis patients are a very heterogeneous group but here we present a homogeneous cohort with the most severe cases of pneumococcal sepsis; untreated on admission, all rapidly developing septic shock. These patients represent a particular diagnostic challenge, having a very narrow 'therapeutic window' and a devastating course if diagnosis is delayed.

\section{Conclusion}

We found it concerning that the qSOFA criteria missed the diagnosis on admission in nearly one-third of the patients. Both SIRS criteria $\geq 2 / 4$ and $\geq 3 / 4$ identified more patients, although only SIRS criteria $\geq 2 / 4$ reached statistical significance compared to qSOFA $\geq$ $2 / 3$. The combination of qSOFA and SIRS criteria $(\geq 2 / 4$ or $\geq 3 / 4$ ) succeeded in early identification of sepsis in all our patients. Further prospective studies comparing the qSOFA and SIRS criteria in other sepsis populations seem warranted. Until then, it seems prudent to combine qSOFA with both SIRS criteria and good clinical judgment when used as a screening tool in the early phases of severe pneumococcal sepsis.

\section{Acknowledgements}

The authors have not received funding for the study and declare no conflicts of interest. Medical writing experts have not been used.

\section{Sources of Support}

The authors have not received funding for the study and declare no conflicts of interest.

\section{Statement of Equal Authors' Contribution}

All authors have contributed equally in preparing the manuscript.

\section{References}

1. Bone RC, Balk RA, Cerra FB, Dellinger RP, Fein AM, et al. (1992) Definitions for sepsis and organ failure and guidelines for the use of innovative therapies in sepsis. The ACCP/SCCM Consensus Conference Committee. American College of Chest Physicians/Society of Critical Care Medicine. Chest 101: 1644-1655.

2. Levy MM, Fink MP, Marshall JC, Abraham E, Angus D, et al. (2003) 2001 SCCM/ESICM/ACCP/ATS/SIS International sepsis definitions conference. Intensive Care Med 29: 530-538.

3. Singer M, Deutschman CS, Seymour CW, Shankar-Hari M, Annane D, et al. (2016) The third international consensus definitions for sepsis and septic shock (Sepsis-3). JAMA 315: 801-810.

4. Seymour CW, Liu VX, Iwashyna TJ, Brunkhorst FM, Rea TD, et al. (2016) Assessment of clinical criteria for sepsis: For the third international consensus definitions for sepsis and septic shock (Sepsis-3). JAMA 315: 762-774.

5. Raith EP, Udy AA, Bailey M, McGloughlin S, Maclsaac C, et al. (2017) Prognostic accuracy of the SOFA score, SIRS Criteria, and qSOFA score for In-Hospital mortality among adults with suspected infection admitted to the intensive care unit. JAMA 317: 290-300.

6. Freund $Y$, Lemachatti N, Krastinova E, Van Laer M, Claessens YE, et al. (2017) Prognostic accuracy of sepsis-3 criteria for in-hospital mortality among patients with suspected infection presenting to the emergency department. JAMA 317: 301-308.

7. Chen FC, Kung CT, Cheng HH, Cheng CY, Tsai TC, et al. (2019) Quick sepsis-related organ failure assessment predicts 72-h mortality in patients with suspected infection. Eur J Emerg Med 26: 323-328.

8. Trydal E, Martinsen AB, Beisland F, Jacobsen D, Holten AR (2019) Structured evaluation on arrival of patients with sepsis and initiation of antibiotics. Tidsskr Nor Laegeforen 139.

9. Askim A, Moser F, Gustad LT, Stene H, Gundersen M, et al. (2017) Poor performance of quick-SOFA (qSOFA) score in predicting severe sepsis and mortality - a prospective study of patients admitted with infection to the emergency department. Scand J Trauma Resusc Emerg Med 25: 56.

10. Mellhammar L, Wullt $S$, Lindberg A, Lanbeck $P$, Christensson B (2016) Sepsis incidence: A population-based study. Open Forum Infect Dis 3: ofw207.

11. Williams JM, Greenslade JH, McKenzie JV, Chu K, Brown AF (2017) Systemic inflammatory response syndrome, quick sequential organ function assessment, and organ dysfunction: Insights from a prospective database of ED patients with infection. Chest 151: 586-596.

12. Churpek MM, Snyder A, Han X, Sokol S, Pettit N, et al. 
(2017) Quick sepsis-related organ failure assessment, systemic inflammatory response syndrome, and early warning scores for detecting clinical deterioration in infected patients outside the intensive care unit. Am J Respir Crit Care Med 195: 906-911.

13. Rhodes A, Evans LE, Alhazzani W, Levy MM, Antonelli M, et al. (2017) Surviving sepsis campaign: International guidelines for management of sepsis and septic shock: 2016. Intensive Care Med 43: 304-377.

14. Seymour CW, Gesten F, Prescott HC, Friedrich ME, Iwashyna TJ, et al. (2017) Time to treatment and mortality during mandated emergency care for sepsis. N Engl J Med 376: 2235-2244.

15. Ferrer R, Martin-Loeches I, Phillips G, Osborn TM, Townsend S, et al. (2014) Empiric antibiotic treatment reduces mortality in severe sepsis and septic shock from the first hour: Results from a guideline-based performance improvement program. Crit Care Med 42: 1749-1755.

16. Liu VX, Fielding-Singh V, Greene JD, Baker JM, Iwashyna TJ, et al. (2017) The timing of early antibiotics and hospital mortality in sepsis. Am J Respir Crit Care Med 196: 856863.

17. Rodriguez RM, Greenwood JC, Nuckton TJ, Darger B, Shofer FS, et al. (2018) Comparison of qSOFA with current emergency department tools for screening of patients with sepsis for critical illness. Emerg Med J 35: 350-356.

18. Ursin Rein P, Jacobsen D, Ormaasen V, Dunlop O (2018) Pneumococcal sepsis requiring mechanical ventilation: Cohort study in 38 patients with rapid progression to septic shock. Acta Anaesthesiol Scand 62: 1428-1435.
19. Bernard GR, Vincent JL, Laterre PF, LaRosa SP, Dhainaut JF, et al. (2001) Efficacy and safety of recombinant human activated protein $\mathrm{C}$ for severe sepsis. N Engl J Med 344: 699-709.

20. Vincent JL, Bernard GR, Beale R, Doig C, Putensen C, et al. (2005) Drotrecogin alfa (activated) treatment in severe sepsis from the global open-label trial ENHANCE: Further evidence for survival and safety and implications for early treatment. Crit Care Med 33: 2266-2277.

21. Brun-Buisson C, Meshaka P, Pinton P, Vallet B, Group ES (2004) EPISEPSIS: A reappraisal of the epidemiology and outcome of severe sepsis in French intensive care units. Intensive Care Med 30: 580-588.

22. FB Taylor Jr, CH Toh, WK Hoots, H Wada, M Levi, et al. (2001) Towards definition, clinical and laboratory criteria, and a scoring system for disseminated intravascular coagulation. Thromb Haemost 86: 1327-1330.

23. Simpson SQ (2018) SIRS in the Time of Sepsis-3. Chest 153: $34-38$.

24. Simpson SQ (2016) New sepsis criteria: A change we should not make. Chest 149: 1117-1118.

25. Haydar S, Spanier M, Weems P, Wood S, Strout T (2017) Comparison of QSOFA score and SIRS criteria as screening mechanisms for emergency department sepsis. Am J Emerg Med 35: 1730-1733.

26. Johansen K, Hansen ST Jr (1993) Symmetrical peripheral gangrene (purpura fulminans) complicating pneumococcal sepsis. Am J Surg 165: 642-645. 